\title{
UNA REFLEXIÓN CRÍTICA DE LA FUNDAMENTACIÓN FILOSÓFICA DE LAS PRÁCTICAS PEDAGÓGICAS
}

A critical review of the philosophical basis of pedagogical practices ${ }^{14}$

Alfonso Paz Samudio*

Paz, A. (2016). Una reflexión crítica de la fundamentación filosófica de las prácticas pedagógicas. Revista PACA, 8, 75-95

\section{Resumen}

En este trabajo se busca dar una fundamentación filosófica adecuada al concepto de práctica pedagógica. La fundamentación filosófica se presenta frecuentemente para las teorías pedagógicas completas, no para aspectos aislados como el tema que abocamos. Por eso, postulamos un marco teórico complejo con ocho categorías: tres de la vida activa (labor, trabajo, acción), tres de la vida del espíritu (pensamiento, voluntad, juicio) y dos de la imaginación (imaginación radical individual, imaginación colectiva instituyente). Este esquema se basa fundamentalmente en Castoriadis y Arendt, que convergen en una problemática común esbozada por Kant. En ese orden de ideas, las prácticas pedagógicas hacen parte de la acción analizada por Arendt y clarificada por Lobo. La lógica que se sigue es la dialógica en que se comparan, desde el marco teórico, los diversos puntos

\footnotetext{
14"CONSTRUCCIÓN DE LOS CONCEPTOS BÁSICOS DE LA MATEMÁTICA FUNDAMENTAL MEDIANTE LAS TIC", Convocatoria interna de la DGI de la USC, marzo de 2018 a Septiembre de 2019; alpaz@usc.edu.co; investigación financiada por la USC con participación de docentes y estudiantes. Líder: Dr. Alfonso Paz Samudio; colíder: Mg. Fernando Giraldo Montero

*Doctor en Educación. Docente investigador de TC de la Universidad Santiago de Cali.

Email: alpasamudio@gmail.com
} 
de vista y se distinguen los aciertos y los puntos débiles, para esbozar una respuesta aproximada. Como criterios se utilizan los principios de la hermenéutica, la coherencia y adecuación al contexto, pero también la concordancia con los datos de que se disponga. La aplicación de los puntos de vista de Arendt ayuda a detectar puntos neurálgicos de los conceptos de práctica pedagógica, pero también permite esbozar problemas como la incorporación del perdón en la acción pedagógica y cuál sería el sentido de una pedagogía del amor.

Expresiones claves: Prácticas pedagógicas, acción, labor, trabajo

\begin{abstract}
:
This paper seeks to provide a philosophical foundation appropriate to the concept of pedagogical practice. The philosophical foundation is often presented for complete pedagogical theories, not for isolated aspects such as the topic we are addressing. For that reason, we postulate a complex theoretical framework with eight categories: three of the active life (work, action), three of the life of the spirit (thought, will, judgment) and two of the imagination (individual radical imagination, collective imagination instituting). This scheme is based fundamentally on Castoriadis and Arendt that converge on a common problem outlined by Kant. In that order of ideas, the pedagogical practices are part of the action analyzed by Arendt and clarified by Lobo. The logic that is followed is the dialogic in which, from the theoretical framework, the different points of view are compared and the successes and weak points are distinguished, in order to outline an approximate answer. As criteria, the principles of hermeneutics, coherence and adaptation to the context are used, but also the agreement with the available data. The application of the points of view of Arendt helps to detect neuralgic points of the concepts of pedagogical practice, but also allows to outline problems like the incorporation of the pardon in the pedagogical action and which would be the sense of a pedagogy of the love.
\end{abstract}

Key expressions: Pedagogical practices, action, labor, work 


\section{Introducción}

Esta indagación busca fundamentar el concepto de prácticas docentes desde los puntos de vista de H. Arendt acerca de la acción. Se parte de que las referencias a las prácticas docentes desde lo cognitivo, lo antropológico, lo sociocultural, las experiencias nacionales e internacionales deben ser complementadas con un enfoque filosófico de la acción, ya que muchas descripciones de la expresión "prácticas pedagógicas" actualmente son el comienzo de teorizaciones sobre la práctica que la sustituyen indebidamente. Los referentes bibliográficos son Bubner (1984) y Arendt (2005).

La fundamentación filosófica se presenta generalmente para las teorías pedagógicas, no para aspectos aislados como el tema que abocamos. Es decir, la fundamentación filosófica se presenta para un enfoque educativo o pedagógico de manera holística, no particular. Ver (Ross, 2013), (Matthews, 2012), (Haave, 2014), Morton and Guerin, 2017) y (Himes and Schulenberg,2013).

Entonces, la pregunta clave de investigación podría formularse así:

¿Cómo fundamentar filosóficamente de manera adecuada las prácticas pedagógicas?

Por eso, este trabajo busca este objetivo específico:

- Dar una fundamentación filosófica de las prácticas pedagógicas desde la teoría de la acción de Arendt y la crítica del juicio de Kant.

El esquema que sustenta el marco teórico se basa fundamentalmente en Castoriadis y Arendt porque ambos autores parten de una problemática común esbozada por Kant, y asumen una posición crítica consecuente que expone los alcances, pero también los límites de sus indagaciones.

Las referencias sobre la filosofía de H. Arendt y la educación son abundantes pero no se centran en el aspecto particular que examinamos sino en los problemas generales de la educación y la pedagogía que planteó Arendt en 1993. Consultar a (Nieves, 2017), (Díaz y Sánchez, 2008)(Aguilar, 2007), (Enkvist,2009). 
Se trata de una investigación bibliográfica apoyada en autores reconocidos en el análisis y reflexión sobre la problemática propuesta. La lógica que se sigue para dirimir tesis contrapuestas sobre el mismo punto es la dialógica en que se esclarecen, desde el marco teórico, los puntos de vista y se distinguen los aciertos y los puntos débiles, para esbozar una respuesta que recupere los puntos fuertes. Como criterios se utilizan los principios de la hermenéutica, la coherencia y adecuación al contexto, pero también la concordancia con los datos cuantitativos y cualitativos de que se disponga. Mediante estas estrategias se espera alcanzar el logro del objetivo específico y la justificación provisional de la hipótesis anunciada.

\section{Marco teórico}

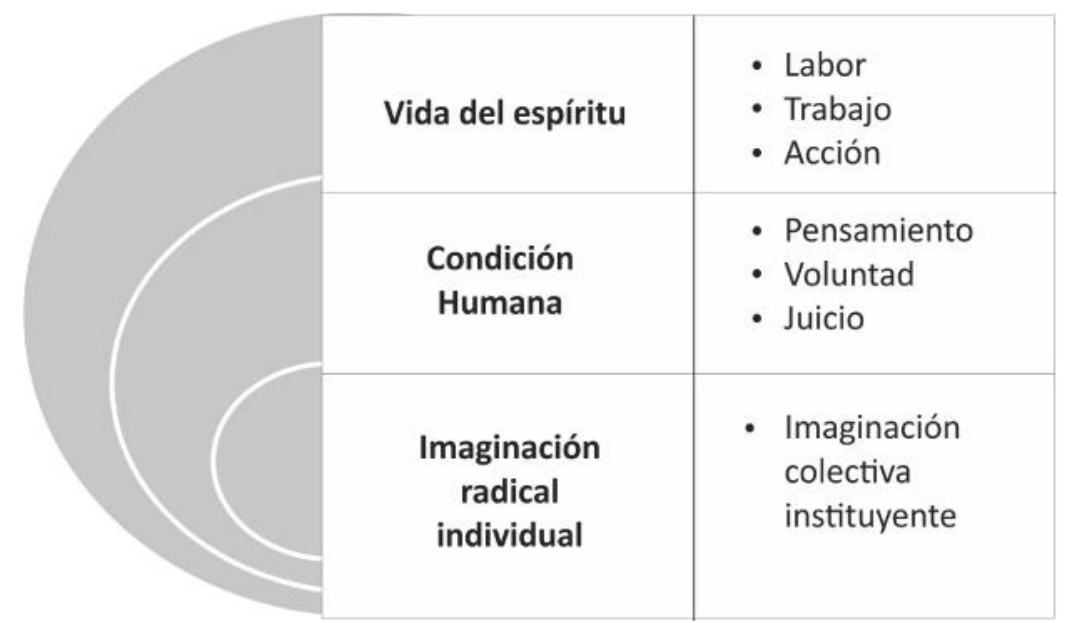

Tabla 1. La imaginación interacciona con la condición humana y con la vida del espíritu,

El esquema que sustenta el marco teórico se basa fundamentalmente en Castoriadis y Arendt porque ambos autores parten de una problemática común esbozada por Kant y asumen una posición crítica consecuente que expone los alcances, pero también los límites de sus indagaciones. El análisis de las obras de Arendt y Castoriadis, con el horizonte de una problemática común esbozada por Kant, es el sustento de nuestro marco teórico ${ }^{15}$. Se espera haber creado una articulación y complementariedad

\footnotetext{
${ }^{15}$ Los lectores interesados en los detalles de este marco teórico, que sirve de sustento al proyecto" Interacciones entre la Sociedad, el conocimiento, las TIC y la Educación Superior", pueden escribirme a alpasamudio@gmail.com.
} 
adecuadas de los planteamientos de Arendt y Castoriadis a las necesidades de investigación formuladas: una articulación y complementariedad de Arendt y Castoriadis, con el sustento de Kant, no puede reducirse a ninguno de los autores que sirven de componentes, ni al autor que sirve de base, ni tampoco elimina completamente ciertas tensiones entre sus puntos de vista. Si ese diálogo fuera imposible, no habría articulación y complementariedad sino simplemente una sopa ecléctica sosa y poco eficaz. Se espera haber superado este riesgo teórico. (Ver Tabla 1).

La imaginación es la capacidad de representar un objeto en la intuición, aun cuando no esté presente. El ser humano individual es un animal radicalmente inepto para la vida. Por eso, la condición de su existencia es la sociedad. En la evolución humana el mundo psíquico, mediante un desarrollo monstruoso de la imaginación, que devino a-funcional, se manifiesta en la insuficiencia y ruptura con las regulaciones instintivas que dominan el comportamiento animal. Está a-funcionalidad se funda sobre dos características de la psique humana: (a) La autonomización de la imaginación, que ya no está funcionalmente sometida. (b) La dominación del placer representativo sobre el placer del órgano (Castoriadis, 1997, p. 182-198).

Hay un estallido del psiquismo animal en el hombre bajo la presión del aumento desmesurado de la imaginación, que deja subsistir elementos importantes de la organización psico-biológica animal, pero también numerosos vestigios de la lógica ensídica (natural) que regula el psiquismo animal. Estos elementos serían totalmente insuficientes para la supervivencia del ser humano individual, pero servirán de soporte a la fabricación por la sociedad del individuo social, es decir, de los humanos. Esta fabricación presupone que la imaginación sensorial persiste más o menos idéntica a través de los especímenes singulares humanos y también que éstos apoyan la imposición de la lógica social. Pero la fabricación social de los individuos a partir de la materia primera que es la psique del recién nacido presupone ya en éste el dominio del placer de representación sobre el placer del órgano. Sin este dominio no habría sublimación posible, ni tampoco vida social. En el acto de hablar, por ejemplo, ya tenemos lo esencial de la sublimación, es decir, el reemplazo de un placer de órgano por un placer que solo tiene relación con la representación. 
A través de esta fabricación social del individuo, la institución somete la imaginación singular del sujeto y solo la deja generalmente manifestarse en y por el sueño, la fantasmatización, la transgresión, la enfermedad.

La condición humana se refiere a lo que hacemos los seres humanos y se identifica con lo que los antiguos denominaron vita activa. Con esta expresión Arendt designó tres actividades fundamentales: labor, trabajo y acción (Arendt, 2005). Mientras que la labor representa las actividades humanas que atienden a las necesidades de la vida, y el trabajo apunta a aquellas actividades en que el ser humano fabrica objetos duraderos a partir de materiales naturales, la acción es el espacio en el cual el hombre desarrolla su capacidad de ser libre, que es su capacidad más notable.

La vida del espíritu se identifica con lo que los antiguos denominaron vita contemplativa. Para Arendt las actividades básicas del espíritu son el pensamiento, la voluntad y el juicio (Arendt, 2002).

Pensamiento y cognición no son lo mismo, según Arendt (2005). El primero es origen de las obras de arte y presente en toda gran filosofía y el segundo, fundamentador de las ciencias. La cognición, por consideraciones prácticas o por "ociosa curiosidad", busca un objetivo definido, y finaliza su proceso una vez alcanzado tal objetivo. El pensamiento no se propone un fin $u$ objetivo, ni siquiera el logro de resultados, aspectos por los cuales se le ha acusado de inútil.

Tanto el pensamiento como la cognición han de distinguirse del razonamiento lógico (deducciones de principios axiomáticos o evidentes, inclusión de casos particulares en reglas generales, o las técnicas de abordar largas y consistentes series de conclusiones). Esta especie de poder mental se parece mucho a la fuerza de labor y suele llamarse inteligencia. El pensamiento busca la comprensión, es decir, el significado del asunto, mientras que el entendimiento persigue a la verdad que es la tarea del intelecto que se comporta como una calculadora determinada por la lógica. Pensar es un diálogo que el sujeto tiene consigo mismo. Una de las sorpresas constitutivas del pensamiento consiste en que problematiza las situaciones humanas. Con el pensamiento se rompe "lo natural" y se trasciende la animalidad con preguntas desafiantes y difíciles como "¿Cuál es la mejor manera de vivir?". La base de estas reflexiones 
sobre pensamiento, cognición y razonamiento lógico es la siguiente cita que establece una distinción que Arendt mantendrá a través de su obra:

Resulta clave para nuestra empresa la distinción kantiana entre Vernunft y Verstand, "razón" e "intelecto"......Kant distinguió entre estas dos facultades mentales tras descubrir el "escándalo de la razón", el hecho de que el espíritu no puede obtener un conocimiento cierto y verificable sobre temas e interrogantes en los que, sin embargo, no puede dejar de pensar. (Arendt, H., 2002, pág. 40)

La facultad de la Voluntad, desconocida para los griegos de la antigüedad clásica y descubierta por los pensadores cristianos, se corresponde con la idea de libertad, ya que la voluntad es la fuente de la acción, como "poder de iniciar espontáneamente una serie de cosas o estados sucesivos" (Kant). El juicio consiste en hacer valoraciones sobre particulares concretos. Las reflexiones inconclusas de Arendt sobre el juicio partieron de Kant. Mientras que el gusto es íntimo, incomunicable y subjetivo, el juicio se ocupa de las cosas del mundo, en donde están los demás.

La división entre Crítica de la razón pura Crítica de la razón práctica implica un abismo infranqueable entre el mundo sensible ( el reino de la naturaleza) y el mundo suprasensible(el reino de la libertad) y se plantea la reconstrucción ordenadora del abismo que tiene que provenir del espíritu, reconstrucción que debe proporcionar la facultad apropiada que haga posible la armonía entre el entendimiento y la razón, y entre la naturaleza y la libertad. Tal facultad va a ser la facultad de juzgar, que en su uso lógico hacía posible el tránsito del entendimiento a la razón, y que ahora va a convertirse en una facultad autónoma con un dominio propio: el de los seres organizados regidos por leyes empíricas, contingentes para nuestro entendimiento. Este ámbito solo puede ser aprehendido por el uso reflexionante de la capacidad de juzgar. Se constituye una filosofía que habría que colocarla entre la filosofía teórica (Crítica de la razón pura) y la filosofía práctica (Crítica de la razón práctica).

Esta nueva esfera (Crítica del juicio) se podría calificar de filosofía teórico-práctica y la contextualizamos en la siguiente tabla adaptada(KANT, 2007:124). 
La facultad de juzgar, en general, es la facultad de pensar lo particular como contenido en lo universal. Si lo universal(la regla, el principio, la ley) es dado, entonces la facultad de juzgar, que subsume lo particular bajo lo universal( incluso si, como facultad trascendental de juzgar, señala a priori las condiciones conforme a las cuales exclusivamente puede ser subsumido bajo aquel universal), es determinante. Pero si sólo es dado lo particular y la facultad de juzgar ha de encontrar el correspondiente universal, entonces ésta es meramente reflexionante. (KANT, 2007: 102-103)

\begin{tabular}{|c|c|c|}
\hline $\begin{array}{c}\text { Facultades totales } \\
\text { del espíritu }\end{array}$ & $\begin{array}{c}\text { Principios } \\
\text { a priori }\end{array}$ & Aplicación \\
\hline $\begin{array}{c}\text { Facultad de conocer } \\
\text { (Entendimiento) }\end{array}$ & Conformidad a leyes & A la naturaleza \\
\hline $\begin{array}{c}\text { Facultad de Juzgar } \\
\text { (juicio - sentimiento de } \\
\text { placer o displacer) }\end{array}$ & Finalidad & Al arte \\
\hline Facultad de desear & Finalidad & A la libertad \\
\hline
\end{tabular}

Tabla 2. (Adaptada de Kant) De las facultades superiores del alma

Si las ciencias naturales y las matemáticas pertenecen al dominio del Entendimiento(conceptos) y la filosofía y el arte a la Razón (ideas), las ciencias sociales, y, por tanto, la pedagogía, ocupan un lugar intermedio entre el entendimiento y la razón, están en el terreno de la Facultad de juzgar y manejan sentidos o significados, pero también tendencias o temas sociales comprobables. Aquí nace la dificultad de diseñar en ciencias sociales marcos teóricos y estrategias metodológicas que sean coherentes, rigurosas y adecuadas, ya que su objeto está en el nomo, no en la Phusis.

Ahora se está en condiciones de justificar las ocho categorías de nuestro marco teórico: tres corresponden a la vida espiritual, tres a la vida activa y dos a la imaginación, que son transversales y omnipresentes en las demás. 
El sistema categorial propuesto forma una red conceptual compleja y abierta que busca establecer los mecanismos ${ }^{16}$ (Bunge,M., 2007) que subyacen a las relaciones y procesos en la educación y la pedagogía.

La perspectiva básica ( a manera de hipótesis regulativa) que anima este trabajo consiste en situarse ideológicamente en la tradición del proyecto de autonomía surgido en la Grecia Clásica Antigua, proyecto que posibilita a la sociedad cuestionarse a ella misma (autonomía, reflexión sobre sí, evaluación y autoevaluación, interrogatorio incondicionado y sin límites).

\section{Estrategias metodológicas}

Se trata de una investigación bibliográfica apoyada en autores reconocidos en el análisis y reflexión sobre la problemática propuesta. La lógica que se sigue para dirimir tesis contrapuestas sobre el mismo punto es la dialógica en que se esclarecen, desde el marco teórico, los puntos de vista y se distinguen los aciertos y los puntos débiles, para esbozar una respuesta que recupere los puntos fuertes. Como criterios se utilizan los principios de la hermenéutica, la coherencia y adecuación al contexto, pero también la concordancia con los datos cuantitativos y cualitativos de que se disponga. Mediante estas estrategias se espera alcanzar el logro de los objetivos específicos y la justificación provisional de las hipótesis anunciadas.

\section{Resultados}

- En este apartado insistiremos en la distinción entre teoría y práctica, como aspectos diferentes en interacción, que no se pueden confundir, pero que examinaremos desde el enfoque de Arendt sobre vita activa y vida del espíritu.

- Se sitúan las prácticas pedagógicas en la categoría de la acción cuya precisión se dificulta por emplear analogías insuficientes con la causalidad y el significado.

\footnotetext{
${ }^{16}$ Según Bunge, el modelo más simple de un sistema material (que pertenece a uno de los siguientes niveles: físico, químico, biológico, social o tecnológico) es la lista de su composición, entorno, estructura y mecanismo(s). Los mecanismos son los procesos característicos que hacen del sistema material lo que esy lo cambian de la manera peculiar en que el sistema cambia (Bunge, 2007:182-208).
} 
- Con Bubner (1981) se podrían definir las acciones como logros. Un logro es la realización sistemática de todo lo que trata una acción, es decir, el alcance pleno de sus objetivos o metas. Si uno se refiere a la práctica como logro, la demarca de otro tipo de actividad: poiesis o producciòn. La práctica es estructuralmente distinta de esos procesos que se dirigen hacia la producción de un objeto externo a ellos mismos. Las reglas para la práctica en un contexto de acción subjetiva del individuo se denominan máximas. Las reglas intersubjetivas que se establecen para la acción colectiva en grupos o en la sociedad como un todo se denominan normas.

- La razón práctica siempre es razón concreta y gira en torno de casos particulares. Por eso, una filosofía que tiene que ver con lo práctico debe partir de que es simplemente teoría, y que cualquier cosa que tenga que ver con la práctica no se encuentra nunca con los mismos estándares de perfección y exactitud con los que operan las ciencias puramente teóricas. Todo lo práctico implica un problema de concreción. Las teorías solo pueden ofrecer generalidades pero la acción, sin embargo, tiene lugar aquí y ahora. Existe el peligro de sustituir la práctica por una construcción teórica o una proyección ideal.

- Una máxima metodológica fundamental de la filosofía consiste en respetar la estructura peculiar de todos los objetos prácticos, lo que implica mantener la frontera entre teoría y práctica. Esta máxima fundamental explica el desacuerdo entre Platón y Aristóteles sobre el conocimiento correcto y la acción correcta. El olvido de esta máxima lleva, por ejemplo, a la creencia, usual hoy en día, de que los problemas prácticos dependen de un supuesto tratamiento científico.

- Para H. Arendt (2005) la cuestión política es una realidad humana que obedece a las capacidades de la acción y el discurso, y esto permite describir su obra a partir de la pregunta por la vita activa y sus aspectos.

- Esta pregunta es contestada en su obra La condición humana que es una reflexión sobre "lo que hacemos" cuyas categorías son labor, trabajo y acción, actividades fundamentales que se corresponden con la vida, la mundanidad y la pluralidad. Todas estas categorías se relacionan de alguna manera con la política, pero la pluralidad (que es la condición de que los hombres vivan en la Tierra y habiten el mundo) es la condición sine qua non de toda vida política.

- Arendt registra, en la vida actual, la victoria del "animal laborans", sujeto de necesidades cuya máxima expresión es la abundancia, sobre 
el hombre libre que tiene la capacidad de la acción y el discurso para transformar la necesidad en libertad y la naturaleza en mundo.

- Por vita activa se entenderán la labor, el trabajo y la acción. Todas las tres actividades y sus correspondientes condiciones están íntimamente relacionadas con la condición más general de la existencia humana: nacimiento y muerte, natalidad y mortalidad. Al ser la acción el escenario privilegiado de la política, la natalidad se constituye en la categoría central del pensamiento político.

- Como todas las cosas con que están en contacto, se convierten inmediatamente en una condición de su existencia, los hombres son seres condicionados. La objetividad del mundo -su carácter de objeto o cosa- y la condición humana se complementan mutuamente.

- Desde las primeras páginas de la obra, H. Arendt clarifica que la expresión "la condición humana" no es equivalente a "la naturaleza humana", basada en una reflexión de San Agustín, a quien parecía imposible contestar la pregunta "¿Qué es el ser humano?". Arendt argumenta que contestar esta pregunta equivaldría "a saltar de nuestra propia sombra", a ser un dios o un semidios. En otras palabras, se cambia la pregunta "¿Qué el ser humano?", cargada de perplejidades por la cuestión más accesible, pero compleja, "¿Cómo es el ser humano?".

\begin{tabular}{|c|c|c|}
\hline Actividad & Descripción & $\begin{array}{c}\text { La condición } \\
\text { humana es }\end{array}$ \\
\hline Labor & $\begin{array}{c}\text { Actividad que corresponde a } \\
\text { la fisiología del ser humano }\end{array}$ & La misma vida \\
\hline trabajo & $\begin{array}{c}\text { Actividad que corresponde } \\
\text { a lo artificial que es una } \\
\text { exigencia del hombre }\end{array}$ & La mundalidad \\
\hline Acción & $\begin{array}{c}\text { Única actividad } \\
\text { interpersonal sin mediar } \\
\text { las cosas o la materia }\end{array}$ & La pluralidad \\
\hline
\end{tabular}

Tabla 3. Actividades de la condición humana

El giro de Arendt hacia la filosofía la lleva a esclarecer la vita contemplativa en sus diversos aspectos: el diálogo del pensamiento consigo mismo, el querer de la voluntad y la valoración de lo real en el juicio. En su libro La vida del espíritu analiza el pensamiento, la voluntad y el juicio pero no 
alcanzó sino a terminar las dos primeras partes y la tercera se reconstruyó y publicó con base en sus borradores y conferencias (1978). Arendt no se pronunció sobre cuál de las dos vita era superior y podría suponerse que consideró que ambas eran importantes y complementarias.

Para Kant, la facultad de juzgar tiene dos acepciones: determinante en que lo universal, en forma de regla, principio o ley, está dado y hay que subsumir lo particular bajo el dominio de lo universal ; reflexionante, en que lo particular es dado y sobre él debe encontrarse lo universal.

Dos perspectivas aparecen en la obra de $\mathrm{H}$. Arendt ante el juicio:

1) la del actor

2) la del espectador imparcial

A partir de ambos, se postula que la facultad de juicio es a la vez:

1) la habilidad fundamental del hombre como ser que actúa.

2) la base de todo pensamiento político. , y

3) una facultad fundamental de la vida de espíritu.

Para H. Arendt el juicio es una de las tres facultades del espíritu humano, distinta del pensamiento y la voluntad. Así el juicio tiende un puente entre la Razón, que maneja ideas y lleva a la filosofía y al arte que son los dominios del pensamiento, y el Entendimiento que maneja conceptos y lleva a las ciencias que son los dominios de la cognición. Por enfrentarse a lo particular para evaluarlo públicamente la facultad de juzgar es una de las más importantes y más difíciles de ejecutar, ya que debe buscar la validez intersubjetiva.

El juicio es una facultad relacional que vincula lo universal con lo particular, y esta capacidad se ve confirmada por sus rasgos básicos: a) intersubjetividad; b) pensamiento ampliado o representativo; c) imparcialidad y, d) carácter dóxico.

En Kant el juicio estaba limitado a la esfera estética. Pero puede extenderse a todo tipo de praxis como lo hizo $\mathrm{H}$. Arendt en la acertada interpretación de Lobo. (Ver Tabla 4).

En todos los ámbitos, el juicio es la facultad que a) se ocupa de lo particular 
qua particular; b) vincula lo particular con lo universal sin que este sea algo dado;c) discierne el universal en el particular, y d) expone su conclusión a la pluralidad en procura de llegar a acuerdos intersubjetivos. En todos los casos, el juicio exige: a)retiro de la participación en la acción (la precede y/o la sigue); b) sustraerse a las condiciones subjetivas, c) conquistarse un punto de vista general haciendo pesar los puntos de vista en el juicio propio, esto es, imparcialidad, mentalidad amplia o pensamiento representativo, y d) comunicabilidad. Todo ello debe producir la validez intersubjetiva general de estos juicios. Existen tensiones estructurales entre los diversos ámbitos de los juicios. Por eso, hay que buscar posibles criterios de articulación. En el caso de los juicios morales, políticos y estéticos estos criterios son: la belleza, la publicidad y la compañía. La posibilidad de alcanzar coherencia y unidad en la vida no evitará los conflictos ni eliminará las tensiones.

\begin{tabular}{|c|c|c|c|}
\hline $\begin{array}{c}\text { Ámbito } \\
\text { del Juicio }\end{array}$ & Prioridad & Procura & Perspectiva \\
\hline $\begin{array}{c}\text { Juicio } \\
\text { moral }\end{array}$ & $\begin{array}{c}\text { Integridad } \\
\text { del Yo }\end{array}$ & La acción propia & Acción \\
\hline $\begin{array}{c}\text { Juicio } \\
\text { político }\end{array}$ & $\begin{array}{c}\text { Integridad } \\
\text { del mundo }\end{array}$ & La acción concertada & $\begin{array}{c}\text { Actor } \\
\text { político }\end{array}$ \\
\hline $\begin{array}{c}\text { Juicio } \\
\text { histórico }\end{array}$ & $\begin{array}{c}\text { Significado } \\
\text { del pasado }\end{array}$ & $\begin{array}{c}\text { Comprensión y } \\
\text { reconciliación }\end{array}$ & Espectador \\
\hline $\begin{array}{c}\text { Juicio } \\
\text { estético }\end{array}$ & $\begin{array}{c}\text { Apariencia } \\
\text { del mundo }\end{array}$ & $\begin{array}{c}\text { Complacencia inactiva } \\
\text { de la contemplación }\end{array}$ & Espectador \\
\hline $\begin{array}{c}\text { Juicio } \\
\text { pedagógico }\end{array}$ & Formación & Autonomía & Actor \\
\hline
\end{tabular}

Tabla 4. Adaptada de María Fátima Lobo, ampliada por A.P.S

\section{Discusión}

La insistencia en distinguir entre teoría y práctica es importante porque circulan textos en que esta distinción se borra injustificadamente. EI MEN en uno de sus materiales, La práctica pedagógica como escenario de aprendizaje, plantea esta descripción: 
En este marco, la práctica pedagógica se concibe como un proceso de auto reflexión, que se convierte en el espacio de conceptualización, investigación y experimentación didáctica, donde el estudiante de licenciatura aborda saberes de manera articulada y desde diferentes disciplinas que enriquecen la comprensión del proceso educativo y de la función docente en el mismo. Este espacio desarrolla en el estudiante de licenciatura la posibilidad de reflexionar críticamente sobre su práctica a partir del registro, análisis y balance continuo de sus acciones pedagógicas, en consecuencia, la práctica promueve el desarrollo de las competencias profesionales de los futuros licenciados (MEN, p.5).

En síntesis, la práctica pedagógica se concibe como un proceso de autorreflexión, es decir, se equiparan práctica y teoría filosófica con lo cual se crea innecesariamente una confusión.

Pero esta distinción entre teoría y práctica no significa separación, sino interacción. Como la razón práctica es razón concreta y gira en torno de casos particulares, entonces una teoría sobre la práctica es una guía para la acción y su lógica es la del juicio cuyo objeto es lo particular como particular.

Hay una diferencia esencial entre acción (praxis) y trabajo (póiesis) que Arendt insiste en mostrar. Mediante el trabajo, los seres humanos, al unir el saber teórico y el dominio práctico requerido (techné), son capaces de fabricar entes artificiales que quedan en el mundo y lo constituyen. En el trabajo los hombres son capaces de producir realidades distintas de sí mismos, mientras que en la acción no se produce nada tangible distinto de sí mismo .De manera que la naturaleza de la acción y del discurso no admite ser explicada en términos instrumentales, como subordinada a un propósito exterior a la propia actividad, es decir, la naturaleza de la acción es performativa. Lo que sí produce la acción son relaciones e historias. Al actuar los hombres interactúan unos con otros más allá de todo propósito material o utilitario.

Aunque la acción y el discurso están sometidas al principio de las consecuencias no deseadas, estarían entre aquellas actividades que Aristóteles llamó energeia, es decir: 1) no persiguen un fin; 2) no dejen un producto tras sí; $y, 3$ )su significado radica enteramente en la pura actuación. 
Por tanto, son un fin en sí mismas y se sustraen a la categoría de medios y fines. Por tanto, las acciones y palabras de los hombres deben juzgarse por su virtuosismo y grandeza, que solamente se aprecian en la actuación. En Arendt, la inmanencia de la acción tiene dos modos de justificación. En el primero, el sujeto es agente y paciente en virtud de la inmanencia del fin en praxis y lexis (El acto enfocado ontológicamente). En el segundo, el sujeto es agente y paciente porque sus actuaciones acontecen en el mundo común al que afectan, con lo que provocará reacciones que tornarán al sujeto y lo afectarán. El primer sentido nos revela el poder realizador de la acción, de su riqueza y dignidad. El segundo nos remite a su fragilidad y contingencia. Entonces la acción, que en tanto initium es señal de renovada natalidad, resulta por lo mismo contingente, impredecible, irreversible, lo cual caracteriza al ámbito de los asuntos humanos que se distinguen por su fragilidad y contingencia.

Frente a la irreversibilidad y la impredectibilidad de la acción, Arendt postula la capacidad de perdonar y la de hacer promesas. El poder de perdonar es una posibilidad de redención frente a los daños que nuestras acciones y palabras irreversibles pudieran causar. Como tal es una de las virtualidades de la propia acción. Perdonar es un modo eminente de acción, un acto de libertad que libera, ya que impide que quedemos condenados a las consecuencias de un solo acto. Para Arendt, el perdón: 1) es un poder y una necesidad específicamente humana tanto de recibirlo como de darlo; 2) sus supuestos son la pluralidad y la mundanidad propiamente humanas; por eso, su carácter estrictamente interpersonal; 3) originariamente tiene la forma de don; 4) su razón de ser está en la falta de soberanía del actor, y 5) con el perdón los hombres se liberan de la lógica reactiva de la venganza. Arendt desvincula el perdón del amor, ya que el amor es una relación extraordinaria que pertenece por naturaleza a la vida privada. Para Arendt el amor es una fuerza poderosa pero completamente antipolítica: impulsa el abandono del mundo, de su publicidad y pluralidad, para realizarse en la intimidad de los amantes.

Las anteriores reflexiones plantean nuevos e interesantes problemas sobre el perdón, la capacidad de hacer promesas y el amor en los asuntos pedagógicos. 


\section{A manera de conclusión}

Al presentar un marco teórico híbrido se debe aclarar que Arendt acepta la imaginación como una facultad básicamente representativa de lo que está ausente para los sentidos. Es decir, la imaginación transforma un objeto visible en una imagen invisible permanente. Por eso, el pensamiento, gracias a la imaginación y a la memoria, goza de cierta libertad mental respecto del tiempo y del espacio y se permite anticipar el futuro, reflexionarlo como si estuviera presente y recordar el pasado como si no hubiera desaparecido. La imaginación es conditio sine qua nom para los cambios que nuestras acciones buscan producir en la realidad. Estas acotaciones de Arendt están en el horizonte intelectual de Kant. Lo que hace Castoriadis es radicalizar la categoría de la imaginación hasta constituirla como la categoría central. Castoriadis constata que la imaginación humana es disfuncionalizada, liberada del imperativo biológico y sus finalidades, con la capacidad de crear formas y contenidos que no dependen de ninguna necesidad, pero apoyada en la dimensión animal de lo humano. Este dato primario de la inserción individual es modelado y domeñado por la socialización pero nunca completamente. Es la interacción entre imaginación radical individual e imaginario colectivo instituyente la que permite comprender lo que acontece a una sociedad y a sus integrantes.

El marco teórico acepta esta primacía de la imaginación y considera que las categorías de la vida activa y de la vida del espíritu se ven afectadas por esta primacía sin perder su relativa autonomía y su necesaria complementariedad.

Recientemente H. Harari (2015) ha escrito De animales a dioses en que plantea una nueva visión de la historia humana, con una escala cronológica que se inicia en el Big Bang y un enfoque evolucionista enriquecido con aportes de diversas ciencias. Harari ${ }^{17}$ ofrece en esta obra una

\footnotetext{
${ }^{17}$ Posiblemente Harari, que es un joven y brillante historiador hebreo, no conoce la obra de Castoriadis, ya que este autor no aparece referenciado en su bibliografía. Lo importante consiste en que Harari ilustra de manera magistral cómo la imaginación radical individual y el imaginario social instituyente construyen ficciones compartidas (leyendas, mitos, dioses, religiones, grandes corporaciones, imperios, naciones, dinero que unen grandes colectividades.
} 
caracterización del lenguaje humano que confirma, de manera novedosa, el enfoque de Castoriadis sobre la imaginación radical individual y el imaginario social instituyente. Según Harari entre hace 70.000 y 30.000 años aparece una revolución cognitiva que se manifestó en nuevas formas de pensar y comunicarse, y la evidencia de la invención de barcas, lámparas de aceite, arcos, flechas, agujas, objetos de arte y joyería.. El corazón de esta revolución cognitiva es un lenguaje flexible, que da información acerca del mundo y de nosotros (chismorreo), pero con la capacidad de transmitir acerca de cosas que no existen en la realidad (leyendas, mitos, dioses y religiones) que se comparten colectivamente. Esta capacidad de compartir ficciones mantiene unidas grandes colectividades (ciudades, imperios y grandes corporaciones, etc.) ya que el lenguaje directo y el chismorreo pueden mantener hasta 150 personas asociadas, pero después de este límite, establecido por la sociología, el colectivo colapsa (Harari, 2015: 33-54).

El sentido de la expresión prácticas pedagógicas es plural: se podría interpretar como la totalidad de los haceres respecto a la pedagogía sin tener en cuenta la distinción que plantea Arendt en labor, trabajo y acción. (Ver Díaz, 2001:16-1). Otros autores hacen depender este sentido de la investigación (Sosa, 2014). En Colombia es notable el programa de investigaciones del Grupo de Historia de la Práctica Pedagógica en Colombia, y nos atreveríamos a afirmar que el enfoque de este grupo da una primacía a la acción pedagógica pero su mirada está bajo la influencia de Foucault (Zuluaga, 1999). Ahora bien, como estamos en el terreno de los significados o sentidos, no se pretende que haya puntos de vista verdaderos o falsos, sino que simplemente constituyen significados diferentes. Lo que se busca es que las comunidades académicas seleccionen el sentido de las expresiones que van a usar por consenso deliberativo, es decir, que establezcan una validez intersubjetiva, no una verdad absoluta.

En ese orden de ideas, al aceptar las premisas de Arendt sobre el hacer humano situamos las prácticas pedagógicas en la categoría de la acción. Es decir, la práctica pedagógica es la acción pedagógica que tiene como supuesto la pluralidad, es inseparable del discurso y su naturaleza es performativa. La prioridad de las prácticas pedagógicas está en el ámbito de la formación del educando en la autonomía individual y social. Las 
prácticas pedagógicas constituyen actividades interpersonales en que no median las cosas o la materia, y, por eso, no pueden tener carácter instrumental. Como su sentido va más allá de lo utilitario o material, las prácticas pedagógicas producen relaciones e historias.

La acción y el discurso son frágiles porque no pueden predecirse y se someten al principio de consecuencias no deseadas de la acción. Pero a pesar de su fragilidad las sostenemos por ser manifestación de la libertad humana. La inmanencia de la acción se justifica por Arendt de dos maneras.

En la primera, el sujeto es agente y paciente en virtud de la inmanencia del fin en praxis y lexis. En la segunda, el sujeto es agente y paciente porque sus actuaciones acontecen en el mundo común al que afectan, con lo que provocará reacciones que tornarán al sujeto y lo afectarán. El primer sentido nos revela el poder realizador de la acción, su riqueza y dignidad. El segundo nos remite a su fragilidad y contingencia. Entonces la acción, que en tanto initium es señal de renovada natalidad, resulta por lo mismo contingente, impredecible, irreversible, lo cual caracteriza al ámbito de los asuntos humanos en su fragilidad y contingencia.

Frente a la irreversibilidad y la impredectibilidad de la acción, Arendt postula la capacidad de perdonar y la de hacer promesas. El poder de perdonar es una posibilidad de redención frente a los daños que nuestras acciones y palabras irreversibles pudieran causar. Como tal es una de las virtualidades de la propia acción. Perdonar es un modo eminente de acción, un acto de libertad que libera, ya que impide que quedemos condenados a las consecuencias de un solo acto. Arendt desvincula al perdón del amor, ya que el amor es una relación extraordinaria que pertenece por naturaleza a la vida privada. Para Arendt el amor es una fuerza poderosa pero completamente antipolítica: impulsa el abandono del mundo, de su publicidad y pluralidad, para realizarse en la intimidad de los amantes.

Las anteriores reflexiones plantean interesantes y nuevos problemas sobre el perdón, la capacidad de hacer promesas y el amor en los asuntos pedagógicos y cómo incorporarlos en una práctica pedagógica sensata.

Al restringir las prácticas pedagógicas a la acción quedan una serie de actividades pedagógicas que tienen que ver con la labor y el trabajo. 
Las observaciones de John Locke podrían ser muy útiles para delimitar el alcance de la labor en pedagogía. Para Locke que partía de la máxima latina de Juvenal "mens sana in corpore sano", se concentra más en la educación del cuerpo del niño a través del ejercicio para que fortalezca su salud. Este fortalecimiento de la salud atañe a los padres, en primer lugar, y a los maestros en segunda instancia. Ese fortalecimiento implica entrenamiento y disciplina en los niños, desde muy temprana edad, en el ejercicio de las comidas, el uso adecuado de los vestidos, la exposición al frío o al calor, según el caso, para ir creando el hábito de la resistencia corporal al medio circundante. Igual cuidado se extiende con el baño y la higiene de niños y jóvenes. Se trata del fortalecimiento corporal y de la creación de hábitos saludables. Fortalecimiento y hábitos que suponen haceres diarios que tienen que ver con la fisiología, y, por tanto, con la labor. Locke abogaba también porque los jóvenes aprendieran un oficio y se convirtieran en expertos competentes en un arte especial.

En cuanto al trabajo pedagógico, ya sea de carácter intelectual o manual, tiene que ver con todos los procesos de creación o adquisición de conocimientos y con los procedimientos de creación o uso de tecnologías para mejorar la eficacia, eficiencia y productividad del trabajo pedagógico.

¿Cómo denominar a la totalidad de los haceres pedagógicos desde un punto de vista curricular? Nos parece que la denominación debería ser Que hacer Pedagógico, o, Hacer pedagógico, que comprendería las prácticas pedagógicas (en el sentido de Arendt), el trabajo pedagógico y la labor pedagógica.

\section{Referentes Bibliográficos}

- Aguilar Rocha, Samadhi. "La Educación en Hanna Arendt", en Aparte Rei. Disponible en http://serbal.pritic.mec.es/AParteRei/

- Arendt, H. (2005). La condición humana, Barcelona, PAIDOS

- Arendt, H. (2002). La vida del espíritu, Barcelona,PAIDOS

- Arendt, H (1993). Between Past and Future, New York, Penguin Books

- Bubner, Rúdiger (1984). La Filosofía Alemana Contemporánea, Madrid, CÁTEDRA

- Castoriadis, Cornelius (1997). Ontología de la creación, Bogotá, Ensayo\&Error 
- Bunge, Mario (2007). A la caza de la realidad. La controversia sobre el realismo. Barcelona, Gedisa

- Díaz, Mario (1990). " De la práctica pedagógica al texto pedagógico" en Pedagogía y Saberes, No. 1 , 1990, UNIVALLE, p.14-27

- Díaz Quero, Víctor. "Formación docente, práctica pedagógica y saber pedagógico" en Laurus, vol.12,Ext.2006, p.88-103, Caracas, Venezuela. Disponible en http://redalyc.org/articulo.oa?id076109906

- Enkvist, Inger. "Hanna Arendt y la filosofía de la educación" en LA ILUSTRACIIÓN LIBERAL, No.41, 2009, p.51-60

- Haave, Neil. "Six Questions That Will Bring Your Teaching Philosophy into Focus" en FACULTY FOCUS. Higher Ed Teaching Strategies From Magnus Publicatios, JUNE 2ND, 2014

- Harari, Yuval Noah (2015). De animales a dioses, Bogotá D.C., DEBATE

- Himes, Hilleary; Schulenberg, Janet (2013)." Theoretical Reflections: Theory and Philosophy Should Always Inform Practice" en VOICES OF THE CLOBAL COMMUNITY

- Kant, I. (2002).Crítica de la Razón Pura, Madrid, TECNOS

- Kant, I. (2003). Crítica de la Razón Práctica, Buenos Aires, Editorial La Página S.A.Losada

- Kant, I.(2007). Crítica del juicio, Madrid, Austral

- Lobo, María Fátima (2013). Hanna Arendt y la pregunta por la relación entre el pensamiento y la acción, Buenos Aires, BIBLOS

- Locke, John (2012). Pensamientos sobre la Educación, Madrid, AKAL

- Matthews, Michael R. "Philosophical and Pedagogical Problems with Constructivism in Science Education", Tréma, 38, 2012, 40-55 MEN, La práctica pedagógica como escenario de aprendizaje, link: https://www.mineducacion.gov.co/1759/articles-357388_recurso_1.pdf

- Morton, Missy; Guerin. Annie. "Sociocultural Perspectives on Curriculum, Pedagogy, and Assessment to Support Inclusive Education" en The Oxford Research Encyclopedia of Education, Oct.2017

- Nieves Loja, Gerardo Miguel (2017). "Hanna Arendt y el problema de la Educación". Sophia, Colección de Filosofía de la Educación [en línea]. Disponible en <http://www.redalyc.org/articulo.oa?id=441852191008>

- Nixon, Jon. (2015). "Hanna Arendt and Education: Broadening the Debate", en Prospero: A Journal of New Thinkingin Philophy for Education and Cultural Continuity, Volume 21, Issue 3, 2015, p.

- Ross, Alexandria (2013). "Reflective Pedagogy Practice: Philosophy of Education" en LEHMAN COLLEGE, CUNY, November 4, 2013 
- Sosa León, Aura Teresa (2014). "La práctica pedagógica, una mirada desde la investigación", ponencia en el Congreso Iberoamericano de Ciencia, Tecnología, Innovación y Educación, Buenos Aires, 12, 13 y 14 de Noviembre de 2014. 